\title{
Faire corps
}

Introduction

\section{Gil Bartholeyns et Frédéric Joulian}

\section{(2) OpenEdition}

Journals

Édition électronique

URL : https://journals.openedition.org/tc/8272

DOI : $10.4000 /$ tc. 8272

ISSN : 1952-420X

Éditeur

Éditions de l'EHESS

\section{Édition imprimée}

Date de publication : 1 décembre 2014

Pagination : 4-13

ISBN : 978-2-7351-2346-9

ISSN : 0248-6016

\section{Référence électronique}

Gil Bartholeyns et Frédéric Joulian, «Faire corps », Techniques \& Culture [En ligne], 62 | 2014, mis en ligne le 01 décembre 2017, consulté le 29 septembre 2022. URL : http://journals.openedition.org/tc/ 8272 ; DOI : https://doi.org/10.4000/tc.8272 


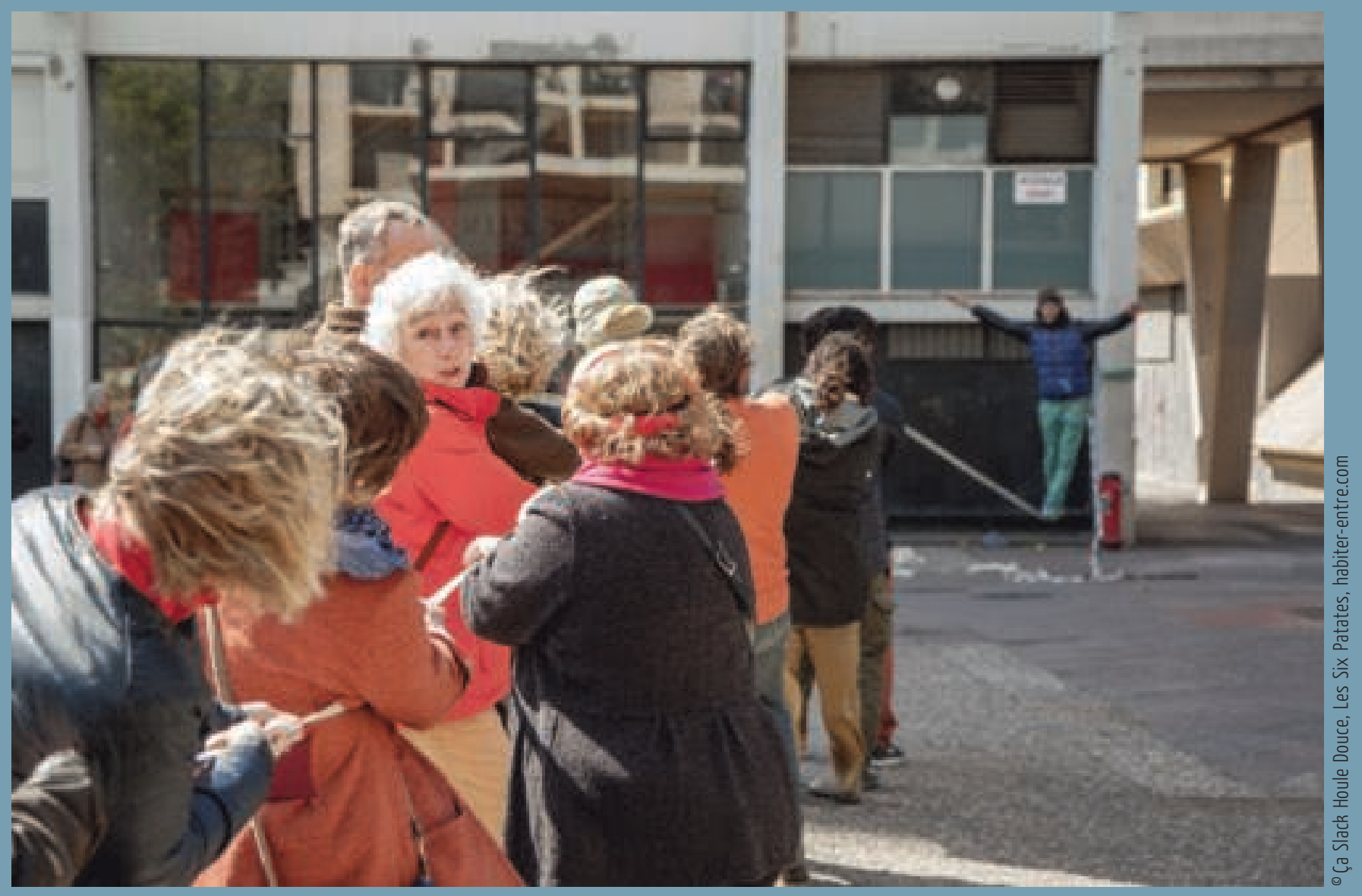




\section{Frédéric Joulian}

EHESS

frederic.joulian@ehess.fr

\section{FAIRE CORPS}

En mémoire de François Sigaut (1944-2012)

«Salut et fraternité»

Quelle ne fut pas notre surprise en recevant par la poste le Dictionnaire de l'historien (2015) auquel nous avons contribué: pas d'entrée «Corps». Le corps n'est pas là. La mode, oui, le vêtement, oui, l'apparence et le geste également. Mais il n'y a rien entre «Corporation» et «Correspondance». Si nous avions su, nous l'aurions faite! Telles ont été notre réaction et celle de nos collègues en constatant que personne n’avait été chargé de rédiger une notice sur le corps.

Comment expliquer cette absence autrement que par un acte manqué? Que la codirectrice de la publication eût elle-même affaire au corps en étudiant la grâce royale qui soustrait le condamné aux supplices corporels, ajoute à ce diagnostic : l'évidence du corps - l'excès peut-être - a conduit à son omission. Car s'il y a bien un objet qui, ces trente dernières années, a conquis les sciences sociales et l'histoire, c'est le corps dans tous ses états. Des grandes entreprises éditoriales comme l'Histoire du corps dirigée par Georges Vigarello, Alain Corbin et Jean-Jacques Courtine (2005-2006) suffisent à en montrer la plénitude.

S'attacher à cette «variable» universelle, médiatrice et irréductible de l'expérience humaine a permis de réunir les chercheurs autour de la conception de la personne humaine (par exemple Godelier \& Panoff 1998, Baschet 2016) ou de son traitement esthétique notamment (cf. Bartholeyns 2010). Une des avancées collectives aura été de tourner définitivement le dos à l'interprétation classique selon laquelle le traitement du corps viserait à arracher l'individu à un hypothétique état de nature pour l'agréger à un collectif, alors qu'il s'agit toujours de le « raccorder» au monde à travers sa propre culture: le marquage, la parure, les décors, toute modification de l'aspect corporel est un mode d'intégration à la fois social et cosmique des individus, dès la naissance et tout au long de la vie. C'est pourquoi 


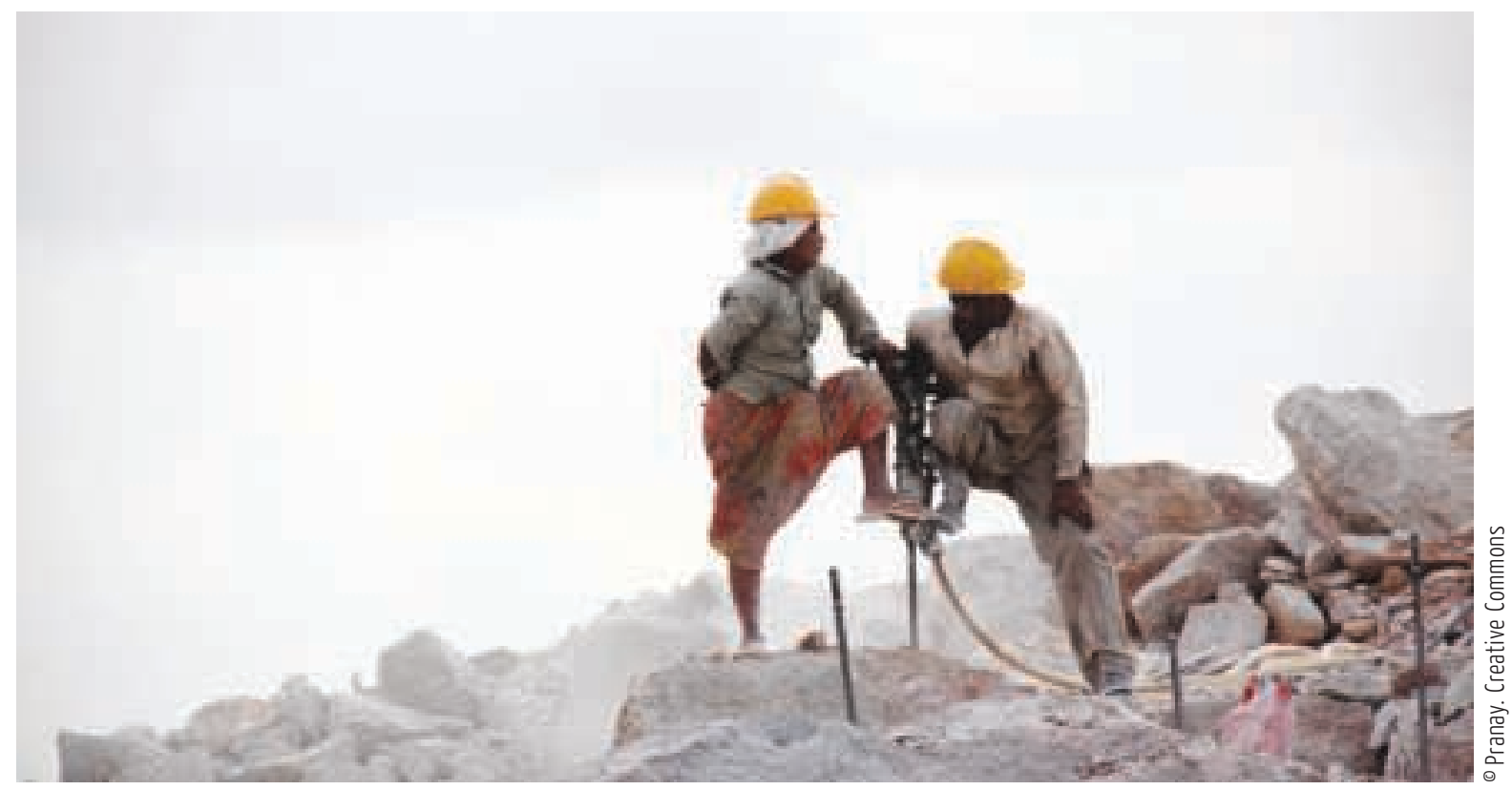

"The way they do it.... ", ouvriers indiens, septembre 2011 Le corps aide la machine et (se) repose sur elle. l'apparence et ses matérialités éclairent si puissamment l'anthropologue et l'historien sur des aspects importants de la cosmologie des sociétés anciennes ou non occidentales (e.g. Taylor \& Viveiros de Castro 2006, Bartholeyns 2009, Lagrou 2016).

Mais les sciences humaines et sociales sont désormais à saturation du corps. «Trop de corps?» demandait en 2009 Bernard Andrieu dans un texte d'indigestion épistémologique (Andrieu 2009). Pourquoi alors ajouter un ouvrage aux ouvrages? Deux constats, au-delà de celui de la qualité de certains travaux en cours, auxquels nous aurions tort de ne pas donner audience, au prétexte d'un sentiment de surabondance largement dépendant du parcours de chacun à travers cette littérature.

Le premier constat est une caractéristique de travaux unanimement reconnus consacrés aux représentations et aux sensibilités corporelles, une caractéristique qui peut être considérée comme un défaut de fabrique. «Pour ces approches», explique Daniel Roche, fortement attaché à une histoire totale mais empirique, «il n'y a plus de questionnement des différenciations sociales, et on ne fait plus jouer les paramètres de distinction et d'intégration des différents milieux socioculturels, ceux qui [sont] acteurs et auteurs du changement, et les autres [...] Quelle est, en quelque sorte, la dynamique des mécanismes de différenciation? On ne se pose plus cette question-là» lorsqu'on entreprend une histoire, par exemple, des pratiques sexuelles, de l'obésité ou de la beauté (Bartholeyns \& Roche 2017: 172). Sans chercher à amender cette historiographie, d'ailleurs largement étrangère à la sociologie du corps, c'est pourtant en mettant les «registres d'appréciations» au cœur du débat que Manuel Schotté a réinterrogé l'« excellence corporelle» par une série d'études parues dans la revue Genèses (Schotté 2016: 3-4). Non seulement la valorisation sociale des personnes par des aptitudes physiques ne va pas de soi, mais les maîtrises corporelles et leurs possesseurs sont perçus différemment selon le cadre de référence (art, sport, spectacle) et selon le milieu social où ces aptitudes se déploient. Ces cadres et milieux vont en outre informer différemment une maîtrise du même type 
(jouer d'un instrument, lutter, ou converser avec brio). Le mode de production (artisanal, industriel...) aura lui aussi une incidence directe sur la valeur de l'opérateur dont le corps est engagé de façon différente selon le processus performatif dominant (vertical, comme la fabrication d'une montre par un seul; horizontal, comme celui de la chaîne de montage) ou selon la division sexuelle du travail (Gardey 2002, Peyrière 2007), avec des conséquences très lourdes pour le corps à l'œuvre (Maitte 2017).

Le deuxième constat est une disparité. Pour dix études consacrées à la construction sociale des corps et de leur perception et à leurs usages sociaux, il y a une étude sur les formes culturelles des facultés du corps, autrement dit «les techniques du corps » et plus largement «les cultures physiques» (Clément 2014). Et pour dix études sur ces «manières traditionnelles» dont les hommes «savent se servir de leur corps» (Mauss 2012 [1935] : 366) et faire société à travers lui, il y a une étude sur le geste technique efficace. En exagérant à peine, nous pourrions poursuivre en disant que pour dix de ces études gestuelles (Harper 1987, ou trente ans plus tard, Bouillon et al. 2017), il y en a une qui aborde le corps, non pas comme outil aliéné ou libéré par la machine (Sennett 2010), mais comme formant un tout avec un artefact en vue d'une action efficace. Ainsi: voler au seul moyen d'un aéronef. Comment? Par délégation de forces contre apports cognitifs. De fait, soit on considère que le corps s'instrumente, se dote de prothèses complexes pour accomplir ce qu'il ne peut accomplir naturellement, soit on pense le corps, la technique, l'outil et le geste de façon holistique et quasi phylogénétique (Sigaut 2012). L'unité corps-instrument, et le corps comme instrument (de bord) de cette unité ont été pensés à raison comme une incorporation de l'artefact en vue d'une synthèse permettant de «faire corps» avec la machine (Warnier 1999: 9 et suivantes), et plus généralement avec l'outil (le marteau...) qui, sans solution de continuité, prolonge en effet notre bras articulé ou celui d'autres grands singes.

De fait, l'une des avancées, malheureusement guère remarquée dans les milieux de l'éthologie ou de la psychologie animale anglo-saxonnes, fut, il y a plus de vingt ans maintenant, d'échapper aux conceptions prothétiques omniprésentes qui faisaient de l'outil le vecteur principal de l'hominisation et l'un des critères discriminants de la définition de l'humanité (Berthelet \& Chavaillon 1993 pour une première ouverture du débat). Comment? En introduisant la vision maussienne totale de la technique et des techniques du corps dans les études naturalistes (Joulian 1996). En l'espèce, Mauss est autant un garde-fou qu'un guide rendant possible l'élaboration, pour la préhistoire et la primatologie, d'un cadre d'analyse structural des conduites humaines et non humaines efficaces (Joulian

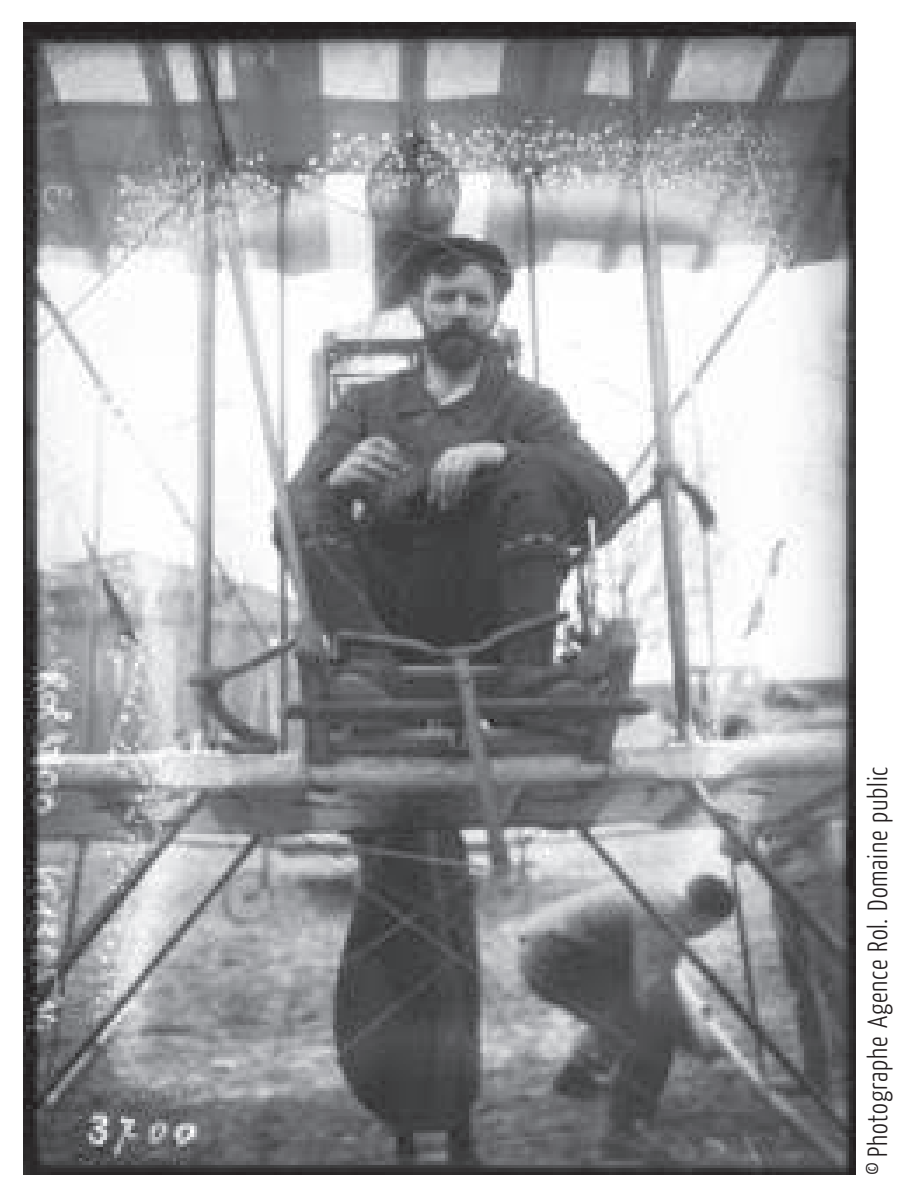


2000); cadre qui permet d'ailleurs de questionner plus avant les limites incertaines des actes humains par les gestes, avant même les objets ou les structures (Joulian 2005, 2009).

Depuis le texte fondateur de Mauss présenté en 1934, maintes fois repris mais si peu approfondi en terme interprétatif (excepté peut-être au sein de l'équipe de Techniques\& Culture, Sigaut 2003, Schlanger 2012), l'anthropologie des techniques du corps se dessine en pointillé. Même chez les anthropologues de la génération suivante comme André-Georges Haudricourt (1948) et André Leroi-Gourhan (1965) (Bert 2010, 2012), ou chez ceux qui dans les années 1970-1980 ont tenté de formaliser les descriptions des gestes (Koechlin 1971), les mouvements (Bril 1984) ou les systèmes techniques (Lemonnier 2010 [1982], Mahias 2004), peu ont suscité l'engouement auquel on aurait pu s'attendre pour un tel programme. D'autres entreprises ethnographiques, comme celle de Kon Wajirô, qui décrit au Japon les habitus posturaux et gestuels dans les années 1920 - révélée ici par l'historien de la culture matérielle, Damien Kunik -, ou comme celle plus proche d'un Junzo Kawada (1980) ou communicationnelles d'un Adam Kendon aux États-Unis (1980) étaient plus systématiques mais elles sont restées isolées. Pourquoi? Nous ne saurions y répondre simplement, mais disons qu'avec les années 1980 la culture reflue des programmes de recherches et avec elle, les gestes culturels et les corps qui les animent.

Or, aujourd'hui, c'est bien d'un tel projet dont nous voulons parler: des corps humains en mouvement, en des séquences d'actions et de gestes dont les accomplissements quotidiens et universels se chargent de multiples significations, se diversifient, se modulent ou se spécialisent selon les activités, en bref «comment les corps font culture».

Ce volume, le dernier de la formule de la revue publiée avec les éditions de la Maison des sciences de l'homme, est né de la réception de plusieurs articles historiques ou sociologiques desquels la question du corps émergeait fortement, et de la sollicitation de travaux en cours sur le corps et le sport au Centre Norbert Elias à Marseille.

Par «corps instrument», et non «corps instrumenté » qui nous aurait davantage orientés vers les seules actions finalisées, nous entendons avec Mauss le corps comme «premier et plus naturel instrument » avec lequel nous agissons sur la matière et l'environnement, ou avec lequel nous interagissons avec nos congénères ou les autres êtres vivants. Nous l'envisageons aussi comme celui que l'on éduque ou cultive dans un contexte ou un autre et qui nous permet de nous socialiser au même titre que (ou avant même) le langage. Ce médiateur universel, aujourd'hui prolongé par maints appendices tactiles et imagés, a principalement attiré des lectures sémiotiques, mettant un peu de côté les approches pragmatiques que certains historiens avaient pourtant eux-mêmes abordés au départ (e.g. Vigarello 1978). Or, les études réunies ici ont en commun de jeter des ponts entre des pragmatiques du corps et d'autres dimensions qui le connotent de plusieurs façons: sociologiques, éthiques, ludiques, heuristiques... Le premier «moment » du livre est ainsi consacré aux questions d'universalité des usages du corps (renvoyant à des universaux biologiques ou culturels); le second est consacré aux usages spécialisés du corps, notamment dans le cas d'apprentissages sportifs ou agonistiques.

La question de l'universalité des conduites humaines s'est longtemps conjuguée à celle d'un malentendu ontologique entre les sciences de la nature d'une part (biologie, physiologie, éthologie, psychologie), à la recherche d'invariants structurels et comportementaux (ceux de l'éthologie humaine par exemple, [Eibl-Eibesfeldt 1972]) et les sciences humaines d'autre part (l'anthropologie et la sociologie en particulier), en quête d'universaux fondés sur les structures mêmes de la cognition humaine (Lenclud 2013, 
Joulian 2015). L'universalité essentialiste n'est toutefois pas l'universalité rationnelle, celle que l'on peut mettre en évidence entre différentes configurations sociales (Taylor 2002); ou celle, pragmatique, que les corps agissants (explicitement ou à leur insu) pourraient idéalement nous permettre de révéler si nous nous mettions à l'ouvrage.

C'est de cette façon qu'Inès Pasqueron appréhende l'universalité du rire: non dans sa généalogie (primates, pré-humains [Van Hoof 1972, Servais 1999]), mais dans la variation interculturelle de ses modes de socialisation et des formes d'apprentissages, que cela soit chez les Nivaclé du Paraguay ou chez les Haya de Tanzanie. Le rire, considéré comme un universel, comme un "propre de l'homme», en réalité s'apprend et se transmet selon les voies les plus diverses. Comme dans l'essai suivant de Philippe Hert, consacré aux savoir (faire) rire des clowns, ou dans celui de Léo Mariani sur la perception des odeurs à travers le temps, un des nœuds complexes à défaire est celui de l'altérité radicale que de telles expressions ou perceptions provoquent sur d'autres corps «cultivés», mis en contact les uns avec les autres. Le rire refoulé des petits enfants nivaclé ne pouvait que surprendre la jeune ethnologue; le décalage de «l'être» clown et du «faire» clown fut pour le sociologue participant, par delà les mots, un moyen de saisir un nouveau paradoxe: celui du corps agissant mis en scène -altérité corporelle que nous retrouvons également avec l'odeur intolérable du durian, qui devient un moyen de questionner l'historicité des «nez» occidentaux et malais du xvII siècle à aujourd'hui.

Ajoutons à ces remarques notre envie de mêler quelque peu des courants d'études trop isolés et de faire se croiser différentes anthropologies, celle des sens, des sensibilités ou des émotions avec celles des objets ou des techniques du corps, «naturellement embarquées» et impliquant un pas réflexif supplémentaire. Rendre compte de la
Orang-outan au zoo de Beauval Par leurs capacités cognitives, les orangs-outans sont capables de se reconnaître dans un miroir, d'agir sur leur corps mais aussi de faire semblant (pretense). Dans cette fin de séquence d'un jeu, le jeune orang-outan joue avec et de son corps, simulant l'immobilisation. II « freeze» ou «fait le mort», dirions-nous pour un jeu d'enfant. La scène illustre à merveille ces « corps instruments», instrumentés par delà la seule matérialité.

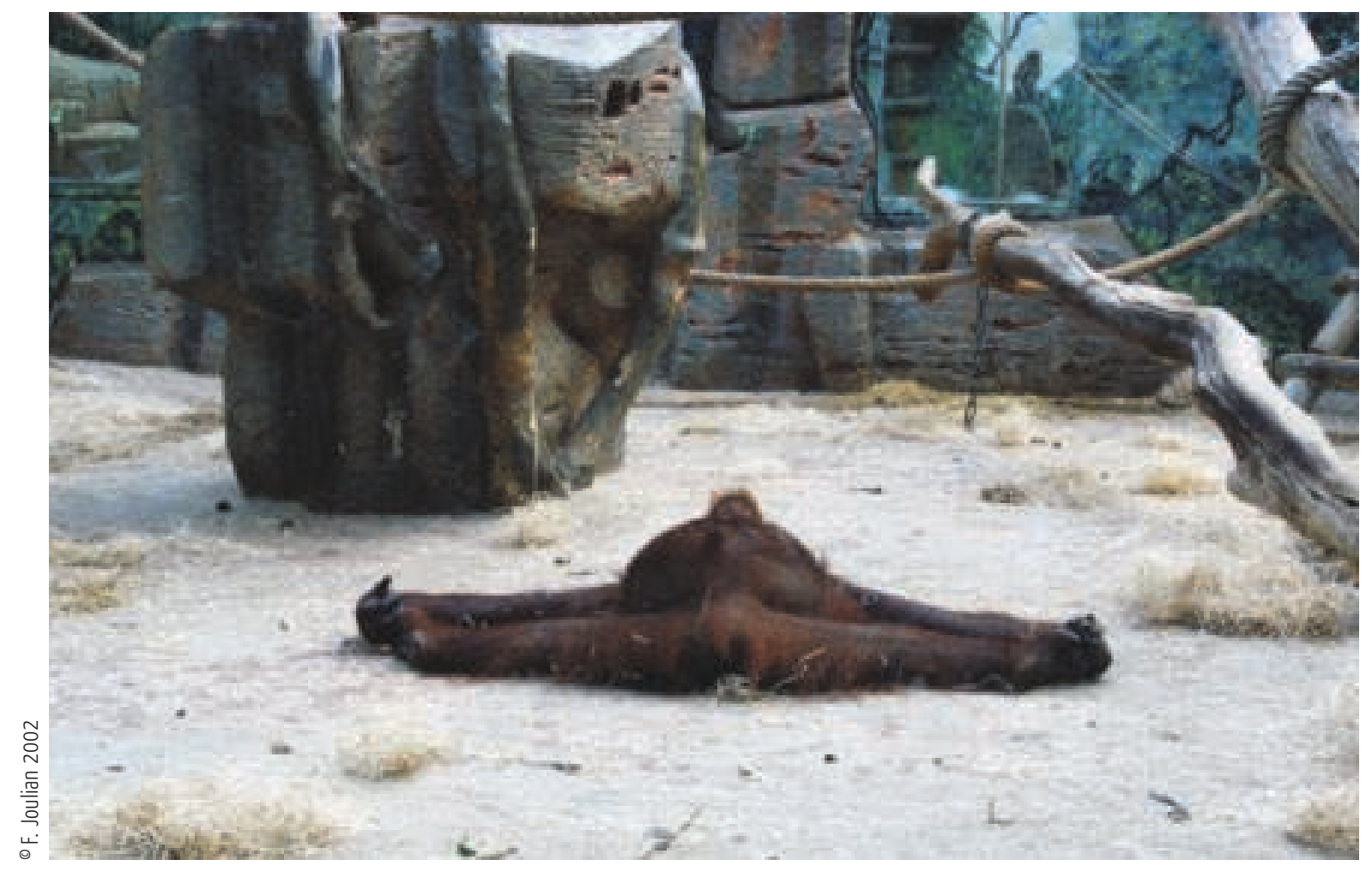


diversité des techniques du corps nécessite en effet, depuis ses débuts, une réflexion sur les cadrages méthodiques de corpus et sur les méthodes de description et d'enregistrement qui furent sans cesse revisités au gré des questionnements et de l'évolution des instruments de captation, depuis André Leroi-Gourhan (1948) ou Claudine de France (1982) à Baptiste Buob (2009) par exemple. À quelle échelle travailler? Les gestes constituent-ils des atomes culturels sur lesquels se fonder? Comment s'articulent-ils à la parole? Les mouvements, processus, séquences opératoires, procès de travail, styles ou performances ne doivent-ils pas être désormais reliés les uns aux autres et non pas analysés séparément comme ce fut majoritairement le cas, et cela dans le cadre de projets scientifiques élargis (cf. Joulian 2016)?

De tels enjeux théoriques et méthodologiques ne sont pas nouveaux. Les gestes et les images, comme outils et objets ethnographiques, ont fait l'objet de diverses approches dont celle dessinée de Kon Wajirô (présentée par Damien Kunik) ou celle rapportée par

Jeune archère « inemuri » dans le train, Kyoto

Quoi de plus universel que dormir ? Mais s'abandonner ainsi en public aux yeux de tous, si naturellement, ne nécessite-t-il pas une confiance en tous, dans un contrôle social, tout à la fois vigilant et indifférent? Une forme « d'être là » et « non là » si étrange au regard occidental (cf. Steger 2016).

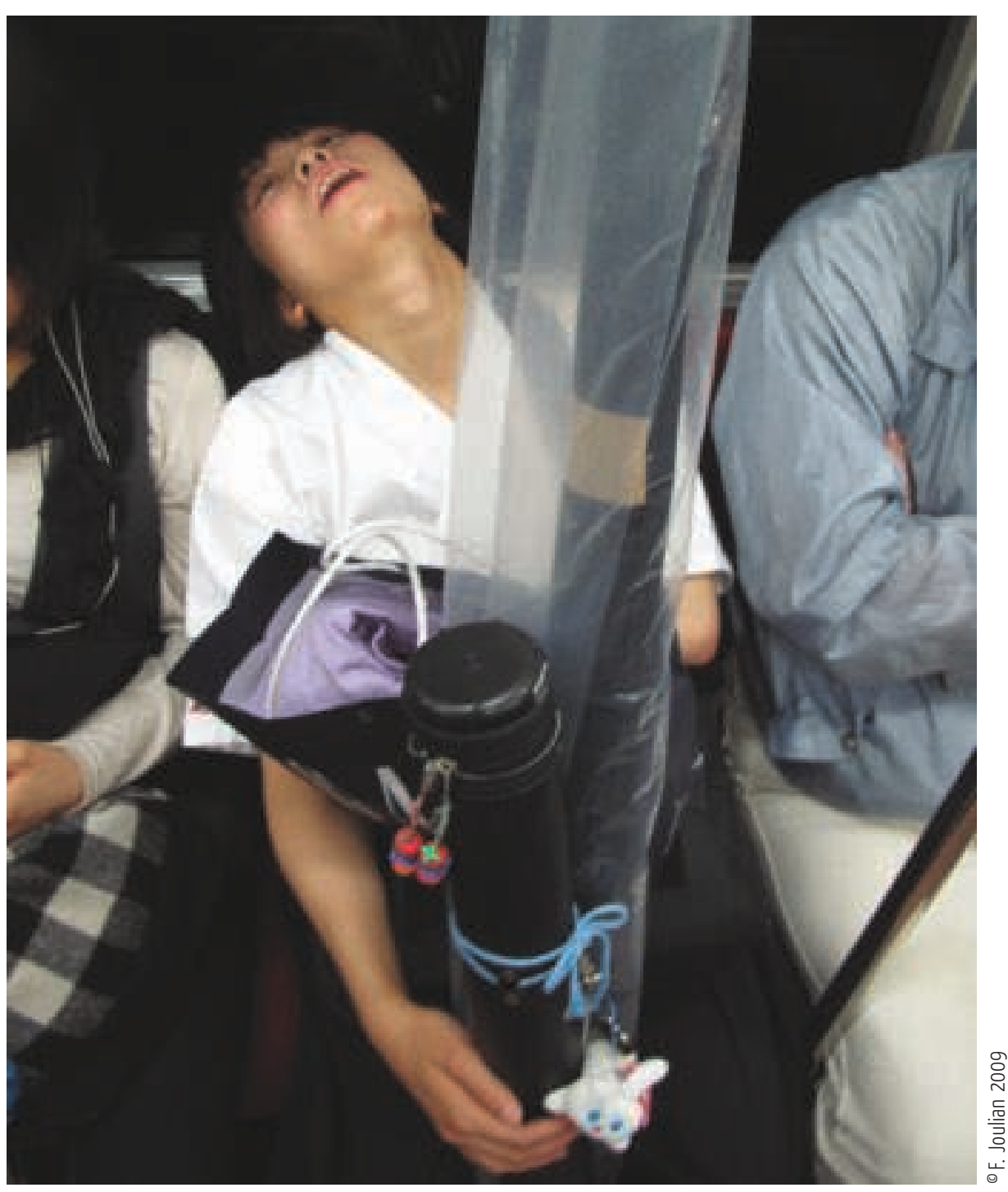


Marie-Claude Mahias qui évoque le travail pionnier de Bernard Koechlin et la création de l'équipe du CNRS Gestes et Images, sorte de sœur synchrone de Techniques\& Culture (1971, 1982). Ces préoccupations descriptives et analytiques se retrouvent sous de nouvelles formes dans l'article d'Éric Vandendriessche sur la cognition pratique mobilisée dans les jeux de ficelles trobriandais et paraguayens, et dans l'étude de Marjolaine Martin sur l'usage généralisé qui est fait de la vidéo par les rugbymen en apprentissage ou par l'auteure elle-même sur le terrain.

La question de l'universalité versus la particularité des techniques du corps des Homo sapiens aurait sûrement nécessité la reprise des travaux sur les traditions animales et l'exploration de la paléontologie des formes gestuelles partagées. Mais cela nous renvoyait à un milieu de références trop éloigné pour maintenir la bonne unité de ce livre. L'ethnographie des sciences réalisée par Frédéric Louchart sur les centres de réintroduction des orangs-outans à Bornéo nous permet toutefois d'approcher les interactions hommes-primates. Dans sa thèse à contre-courant, Louchart envisage les centres de réintroduction comme de nouveaux dispositifs domesticatoires, des lieux où les hommes apprennent ou réapprennent la nature aux anthropoïdes, mais selon des habitus corporels parfois opposés si l'on est dayak, occidental ou orang-outan «acculturé ».

La seconde partie de l'ouvrage correspond à des cultures du corps plus spécialisées, des activités ou des métiers où les normes et les modèles sont réfléchis et parfois transposés dans des règles écrites à usage didactique. Ces savoirs illustrent à quel point les pratiques - guerrières ou sportives notamment - peuvent changer dans l'espace et le temps, de l'escrime médiévale et ses reconstitutions contemporaines selon Sergio Boffa, à l'histoire du basket-ball aux États-Unis et en France, depuis sa naissance dans les années 1890, par Éric Claverie. Nous découvrons également des communautés de pratiques qui se fondent sur la mise en commun des efforts, "solitaires et solidaires», dans le cas du fitness, pour lequel Lilian Pichot décrit des lieux de partage d'épreuves et d'émotions, bénignes dans cet espace, ou malignes dans celui, plus diffus, des jeux et des pratiques guerrières en Afrique Centrale décrits par Thomas Riot, Nicolas Bancel et Herrade Boistelle. De multiples valeurs émanent des corps en action: rationalité et «génie » des nations selon les styles de basket (Claverie); rapidité et agilité des pionniers de la course cycliste ou automobile chez Philippe Gaboriau, des valeurs enchâssées au progrès machinique et politique des premières années du xxe siècle.

À l'invite de François Sigaut (2012), retenons pour finir le rôle si complexe de certains instruments dans les techniques du corps et peut-être la nécessité de ne pas les penser indépendamment des dites techniques, mais avec elles, à l'instar du robot «simulateur » de mêlées décrit par Marjolaine Martin et qui exemplifie non pas un engin autonome et spécialisé, mais un objet intégratif des capacités des «avants» réunis, un engin qui mimique un collectif mais qui est animé par l'entraîneur à l'aide d'un joystick. L'entraîneur dirige non pas un instrument mais plusieurs hommes délégués dans « une mécanique souple» (son expression). En regard, les joueurs qui s'y affrontent sont liés, font « corps », par un engagement physique et des valeurs communes associées au partage et au plaisir.

À n'en pas douter, François Sigaut (1996) et Bob Cresswell (1996) auraient aimé... ce clin d'œil aux instruments les plus divers, et ce coup de chapeau d'un clown-singe aux forces de l'ordre. 


\section{RÉFÉRENCES}

Andrieu, B. 2009 « Trop de corps ? » in C. Doucet et al. dir. Pratiques et usages du corps dans notre modernité. Toulouse : Érès : 7-16.

Bartholeyns, G. 2010 «Faire de l'anthropologie esthétique », in G. Bartholeyns dir. Les apparences de l'homme. Civilisations 59 (2) : 9-40.

— 2009 « L'homme au risque du vêtement. Un indice d'humanité dans la culture occidentale » in G. Bartholeyns, P.-O. Dittmar, M. Har-Peled, V. Jolivet, M. Simon dir. Adam et l'Astragale. Essais d'anthropologie et d'histoire sur les limites de l'humain. Paris : Éditions de la Maison des sciences de l'homme : 99-136.

Bartholeyns, G. \& Roche, D. 2017 « Avis de recherche. Dialogue avec Daniel Roche », Modes pratiques 2 : 171-187. Baschet, J. 2016 Corps et âmes. Une histoire de la personne au Moyen Âge. Paris : Flammarion.

Bert, J.-F. 2012 «Les techniques du corps » de Marcel Mauss. Dossier critique. Paris : Publications de la Sorbonne.

Bert, J.-F. \& A.-G. Haudricourt 2010 Des Gestes aux techniques. Essai sur les techniques dans les sociétés prémachinistes. Paris: MSH-Quae.

Berthelet, A. \& J. Chavaillon dir. 1993 The Use of Tools by Human and Non-human Primates. Symposia of the Fyssen Foundation, vol. 3. Oxford : Oxford Press.

Bouillon, D., Guillerme, A., Mille, M. \& G. Piernas dir. 2017 Gestes techniques, techniques du geste. Villeneuve d'Ascq : Presses universitaires du Septentrion.

Buob, B. 2009 « Filmer, entendre, montrer des savoir-faire. Regards et écoutes croisés dans la médina de Fès », in F. Fogel \& I. Rivoal dir. La relation ethnographique, terrains et textes. Ateliers d'anthropologie 33 [En ligne] http://ateliers.revues.org/8206.

Bril, B. 2010 [1984] « Description du geste technique : quelles méthodes? », TechniquesECulture 54-55 : 242-259. Clément, J. 2014 Cultures physiques. Le rugby de Samoa. Paris : Éditions Rue d'Ulm.

Corbin, A., Courtine J.-J. \& G. Vigarello dir. 2005-2006 Histoire du corps, 3 tomes. Paris : Éditions du Seuil. Cresswell, R. 1996 Prométhée ou Pandore. Propos de technologie culturelle. Paris : Kimé.

France, Cl. de 1982 Cinéma et anthropologie. Paris : Éditions de la Maison des sciences de l'homme.

Eibl-Eibesfeldt, I. 1977 [1972] Éthologie, biologie du comportement, $4^{e} e ́ d$. Jouy-en-Josas : NEB - Ed. scientifiques. Gauvard, Cl. \& J.-F. Sirinelli dir. 2015 Dictionnaire de l'historien. Paris : PUF (Quadrige).

Gardey, D. 2002 « Humains et objets en action : essai sur la réification de la domination masculine » in D. Chabaud-Rychter \& D. Gardey dir. L'engendrement des choses. Des hommes, des femmes et des techniques. Paris : Éditions des archives contemporaines.

Godelier, M. \& M. Panoff 1998 La production du corps. Approches anthropologiques et historiques. Paris : Éditions des archives contemporaines.

Harper, D. 1987 Working Knowledge. Skill and Community in a Small Shop. Chicago : University of Chicago Press.

Haudricourt, A.-G. 1948 « Relations entre gestes habituels, forme de vêtements et manière de porter les charges », Revue de Géographie humaine et d'ethnologie 3 : 58-67.

Joulian, F. 2016 (coordination) « Décrire et enregistrer les techniques du corps », séance de travail du 10 octobre 2016, Axe « Natures, genre, santé », Centre Norbert Elias, La Vieille Charité, Marseille.

— 2015 « André Leroi-Gourhan, Claude Lévi-Strauss, la question de l'unité de l'Homme et de l'évolution » in Ph. Soulier dir. André Leroi-Gourhan, «L'homme, tout simplement». Paris : De Boccard (Travaux de la Maison de l'Archéologie et de l'Ethnologie, René-Ginouvès 20) : 85-101.

— 2005 « Significant Tools and Signifying Monkeys : the Question of Body Techniques and Elementary Actions on Matter Among Apes and Early Hominids » in L. Backwell \& F. d'Errico dir. Proceedings of International Round Table "From tools to Symbols. From Hominids to Modern Humans " / Des outils aux symboles. Des hominidés aux hommes modernes». Johannesburg: Witswatersrand University Press : 52-81.

— 2000 « Techniques du corps et traditions chimpanzières », Les animaux pensent-ils ? Terrain 34 : 37-54 [En ligne] http://terrain.revues.org/document951.html.

— 1996 « Techniques du corps et actions élémentaires sur la matière, avec et sans outil, chez les primates » in F. Sigaut \& G. Lewis dir. Culture and the Uses of the Body, actes du colloque de la Fondation Fyssen, St Germain en Laye, $1^{\text {er-4 }}$ mars 1996.

Kawada, J. et al. 1992 « Les techniques du corps et les caractères morphologiques de deux groupes ethniques 
de l'Afrique Occidentale » in Boucle du Niger. Approches multidisciplinaires. Tokyo : Institut de Recherches sur les Langues et les Cultures d'Asie et d'Afrique, vol. 3 : 125-258.

Kendon, A. 1980 " Gesticulation and Speech : Two Aspects of the Process of Utterance », in M. R. Key dir. The Relation Between Verbal and Nonverbal Communication. La Haye : Mouton : 207-227.

Koechlin, B. 1971 Pour une ethno-technologie. Elément d'un manuel de technologie culturelle (un prototype de grille documentaire), vol. 2, 3. Paris : EPHE-CNRS.

Koechlin, B. \& Lajoux, J.-D. 1982 « Présentation du centre de documentation et de recherche sur la réalité gestuelle des sociétés humaines », Geste et Image, TechniquesE Culture 1 : 131-145.

Lagrou, E. dir. 2016 No caminho da miçanga. Um mundo que se faz de contas, catalogue d'exposition, Rio de Janeiro : Museu do Índio - Funai.

Lenclud, G. 2013 L’universalisme ou le pari de la raison. Anthropologie, histoire, psychologie. Paris : Éditions EHESS / Gallimard / Seuil (Hautes études).

Lemonnier, P. 2010 [1982] « L'étude des systèmes techniques, une urgence en technologie culturelle », in G. Bartholeyns, N. Govoroff \& F. Joulian dir. Cultures matérielles. Anthologie raisonnée de TechniquesECulture. Paris : Éditions de la Maison de sciences de l'homme : 46-67.

Leroi-Gourhan, A. 1965 Le Geste et la Parole, vol. 2. Paris : Albin-Michel.

— 1948 « Cinéma et sciences humaines. Le film ethnologique existe-t-il ? », Revue de géographie humaine et d'ethnologie $3: 42-51$.

Mahias, M.-C. 2004 Le Barattage du monde. Essais d'anthropologie des techniques en Inde. Paris : FMSH Éditions.

Maitte, C. 2017 Colloque Corps au travail. Performance, discipline et fatigue à l'épreuve du métier (XVII $-X X^{e}$ siècle), Université Paris-Est Marne-la-Vallée, 30 juin- $-^{\text {er }}$ juillet.

Mauss, M. 2012 [1935] « Les techniques du corps » in N. Schlanger dir. Techniques, technologie et civilisation. Paris : PUF : 365-394 [Journal de psychologie normale et pathologique 32 : 271-293].

Peyrière M. 2007 « L'emprise de la machine à coudre », Communications 81 (Corps et techniques) : 71-84.

Schlanger, N. 2012 « Une technologie engagée: Marcel Mauss et l'étude des techniques dans les sciences sociales » in N. Schlanger dir. Techniques, technologie et civilisation. Paris : PUF (Quadrige) : 17-134.

Schotté, M. 2016 « Excellences corporelles » in M. Schotté dir. Excellences corporelles, Genèses 103(2) : 3-6.

Sennett, R. 2010 Ce que sait la main. La culture de l'artisanat. Paris : Albin Michel.

Servais, V. 1999 « Autour du chat du Cheshire et de son sourire. Approche comparative du rire et du sourire », L'Homme 39 (150) : 157-175.

Sigaut, F. 2010 [2003] « La formule de Mauss », Efficacité technique, efficacité sociale. TechniquesE Culture 40 (rééd. dans Anthologie raisonnée, TechniquesECulture 54-55 : 357-367).

- 2012 Comment Homo devint faber. Comment l'outil fit l'homme. Paris : CNRS Éditions.

Sigaut F. \& G. Lewis dir. 1996 Culture and the Uses of the Body, Actes du colloque de la Fondation Fyssen, Saint-Germain-en-Laye.

Steger, B. 2016 The Japanese Art of (Not) Sleeping [http://www.bbc.com/future/story/20160506-the-japaneseart-of-not-sleeping].

Taylor, A.-C. 2002 « Invariants et variabilité en anthropologie », in J. Lautrey, B. Mazoyer \& P. Van Geert dir. Invariants et variabilité dans les sciences cognitives. Paris : Éditions de la MSH : 269-287.

Taylor, A.-C. \& E. Viveiros de Castro 2006 « Un corps fait de regards » in S. Breton dir. Qu'est-ce qu’un corps? Paris : Musée du quai Branly / Flammarion : 12-23.

Van-Hoof, J. 1972 « A Comparative Approach to the Phylogeny of Laughter and Smiling » in R. A. Hinde dir. Non verbal communication. Cambridge : Cambridge University Press : 209-241.

Vigarello, G. 1978 Le corps redressé. Histoire d’un pouvoir pédagogique. Paris : J.-P. Delarge.

Warnier, J.-P. 1999 Construire la culture matérielle. L'homme qui pensait avec ses doigts. Paris : PUF.

\section{POUR CITER CET ARTICLE}

Bartholeyns, G. \& F. Joulian 2017 «Faire corps», in G. Bartholeyns \& F. Joulian, Le corps instrument, Techniques \&Culture 62:4-13. 